\title{
Mutation of the TERT promoter leads to poor prognosis of patients with non-small cell lung cancer
}

\author{
SOO-JUNG JUNG ${ }^{1 *}$, DONG-SUN KIM ${ }^{2 *}$, WON-JIN PARK ${ }^{1}$, HYUNSU LEE ${ }^{1}$, \\ IN-JANG CHOI ${ }^{1}$, JAE-YONG PARK ${ }^{3}$ and JAE-HO LEE ${ }^{1}$ \\ ${ }^{1}$ Department of Anatomy, Keimyung University School of Medicine, Daegu 42601; Departments of ${ }^{2}$ Anatomy and \\ ${ }^{3}$ Internal Medicine, School of Medicine, Kyungpook National University, Daegu 41944, Republic of Korea
}

Received March 30, 2016; Accepted December 9, 2016

DOI: $10.3892 / 01.2017 .6284$

\begin{abstract}
Mutations in the promoter region of telomerase reverse transcriptase (TERT) and alterations in telomere length (TL) have been the focus of research in various types of cancer. In the present study, the frequency and clinical characteristics of TERT promoter mutations and TL were studied in non-small cell lung cancers (NSCLC). TERT promoter mutations and TL were analyzed in 188 patients using DNA sequencing and the reverse transcription-quantitative polymerase chain reaction, respectively. The TERT promoter mutation rate was observed to be $2.2 \%$ (4/188 NSCLC cases), and it was significantly associated with regional lymph node invasion $(\mathrm{P}<0.001)$. No significant difference in TL was observed between the patients with and without TERT promoter mutations. TL was decreased in males ( $\mathrm{P}=0.058$ vs. females) and smokers ( $\mathrm{P}=0.008$ vs. non-smokers). Survival analyses demonstrated poor prognoses for patients with NSCLC with TERT promoter mutations $(\mathrm{P}<0.001)$. Multivariate survival analyses demonstrated that TERT promoter mutations were an independent prognostic marker for poor overall survival $(\mathrm{P}=0.045)$. The results of the present study demonstrated that TERT promoter mutation was not frequent in NSCLC; however, it may have value as a prognostic marker in NSCLC.
\end{abstract}

\section{Introduction}

The telomere is a region of repetitive nucleotide sequences at each end of a chromosome. The telomere protects the ends of chromosomes from deterioration or from fusion with adjacent chromosomes. Therefore, telomeres are necessary for

Correspondence to: Jae-Ho Lee, Department of Anatomy, Keimyung University School of Medicine, 2800 Dalgubeoldaero, Dalseo, Daegu 42601, Republic of Korea

E-mail: anato82@dsmc.or.kr

*Contributed equally

Key words: telomerase reverse transcriptase promoter mutation, non-small cell lung cancer, telomere length maintaining genomic integrity and stability (1). Furthermore, telomerase reverse transcriptase (TERT) is a ribonucleoprotein polymerase that maintains telomere ends (2). Activation mutations in the TERT promoter lead to increased expression of telomerase, which sustains telomere length (TL) and genomic stability, thereby allowing cancer cells to continuously divide, and preventing senescence or apoptosis (2). TERT promoter mutations were primarily established in melanoma; however, were subsequently discovered in a number of other common types of cancer, including hepatocellular cancer, bladder cancer, glioblastoma, and thyroid cancer (3-7). In addition, a previous study reported that TERT promoter mutations with ultraviolet signatures are frequent in skin cancer, suggesting that the overexpression of telomerase serves an important role in the pathogenesis of these tumors (2).

Lung cancer is a primary public health problem and a principal cause of cancer-associated mortality worldwide (8). There are two primary types of lung cancer: Small cell lung cancer (SCLC) and non-small cell lung cancer (NSCLC). Between 10 and $15 \%$ of lung cancer cases are SCLC, named for the size of the cancer cells when observed under a microscope (9). By contrast, between 85 and $90 \%$ of lung cancer cases are NSCLC (9). As NSCLC typically grows and spreads more slowly compared with SCLC, it does not frequently respond to chemotherapy, in contrast to SCLC (10). Therefore, there are numerous efforts to develop NSCLC targeted chemotherapy based on oncogenic mutations, including those in epidermal growth factor receptor (EGFR) (10).

However, whether patients with lung cancer exhibit TERT promoter mutations and their prevalence remains unclear due to the low frequency of TERT promoter mutations in lung cancer. In the present study, the presence of TERT promoter mutations in NSCLC was investigated, and the potential association between clinicopathological features and TERT promoter mutations evaluated. To elucidate the role of TERT mutations, TL was also investigated based on previous reports of its prognostic value $(11,12)$.

\section{Patients and methods}

Patients and tissue samples. Tumor specimens and corresponding non-malignant lung tissue specimens $(n=188)$ were provided by the National Biobank of Korea, Kyungpook 
National University Hospital (KNUH; Daegu, South Korea), supported by the Ministry of Health, Welfare, and Family Affairs (Sejong, South Korea). A total of 89 patients were $<60$ years old and 99 patients were $\geq 60$ years old. All materials derived from the National Biobank of Korea, KNUH, were obtained under institutional review board-approved protocols (approval no. KNUMCBIO_14-1010). None of the patients received chemotherapy or radiotherapy prior to surgery.

TERT promoter mutation. Genomic DNA (gDNA) was isolated from tumor samples using QIAamp DNA mini kits (Qiagen, Inc., Valencia, CA, USA). The TERT promoter region was amplified from isolated gDNA using the polymerase chain reaction (PCR) as described previously (13). PCR was performed using AmpliTaq Gold DNA polymerase (Applied Biosystems; thermo Fisher Scientific, Inc., Waltham, MA, USA). The sequences of the forward and reverse primers were 5'-CACCCGTCCTGCCCCTTCACCTT-3' and 5'-GGCTTC CCACGTGCGCAGCAGGA-3', respectively. Thermocycling conditions were 40 cycles of at $94^{\circ} \mathrm{C}$ for $30 \mathrm{sec}, 55^{\circ} \mathrm{C}$ for $30 \mathrm{sec}$ and $72^{\circ} \mathrm{C}$ for $60 \mathrm{sec}$. The PCR products were separated on a $1.5 \%$ agarose gel using electrophoresis and stained with ethidium bromide for 20 mins to confirm the size of the bands. Direct DNA sequencing of the TERT promoter region was subsequently performed using an ABI 3730 DNA sequencer (Bionics Inc., Seoul, South Korea).

Relative telomere length determination. Telomere length was analyzed using quantitative PCR (qPCR) as aforementioned. For the quantitative determination of telomere length relative to nuclear DNA (nDNA), specific primers for telomere (T) and nDNA-encoded $\beta$-globin (S) were selected, according to a previous study (13). The primer sequences were: telomere forward, 5'-GGTTTTTGAGGGTGAGGGTGAGGG TGAGGGTGAGGGT-3' and reverse, 5'-TCCCGACTATCC CTATCCCTATCCCTATCCCTATCC-CTA-3'; $\beta$-globin forward, 5'-CAGCAAGTGGGAAGGTGTAATCC-3' and reverse, 5'-CCCATTCTATCATCAACGGGTACAA-3'. qPCR was carried out using a LightCycler 480 II system (Roche Diagnostics, Basel, Switzerland) with SYBR PCR master mix (Toyobo, Osaka, Japan). Relative telomere length was determined by calculating $\mathrm{T} / \mathrm{S}$ values using the following formula: $\mathrm{T} / \mathrm{S}=2^{-\Delta \mathrm{Cq}}$, where $\Delta \mathrm{Cq}=$ mean $\mathrm{Cq}_{\mathrm{T}}$-mean $\mathrm{Cq}_{\mathrm{S}}(14)$. Each measurement was repeated in triplicate and five serially diluted control samples were included in each experiment.

Statistical analysis. $\chi^{2}$, Fisher's exact test, the Mann-Whitney $\mathrm{U}$ test and Spearman's rank correlation analysis were used to analyze the association between variables using SPSS version 20.0 (IBM SPSS, Armonk, NY, USA). Survival curves, constructed using the univariate Kaplan-Meier estimators, were compared using the log-rank test. Overall survival (OS) was defined as the time between diagnosis and mortality. Disease-free survival (DFS) was defined as the time between diagnosis, and disease recurrence or the development of distant metastasis. Hazard ratios (HRs) and 95\% confidence intervals (CIs) were estimated using a multivariate Cox proportional hazards model, with adjustment for age, gender, EGFR mutation and histological grade. $\mathrm{P}<0.05$ was considered to indicate a statistically significant difference.

\section{Results}

Frequency and clinicopathological characteristics, of TERT promoter mutation and TL in patients with NSCLC. The TERT promoter regions of 188 patients with NSCLC were sequenced and mutations were observed in $2.2 \%(4 / 188)$ of patients. Previous studies reported that C250T (-146C $>\mathrm{T})$ and $\mathrm{C} 228 \mathrm{~T}$ $(-124 \mathrm{C}>\mathrm{T})$ were points of frequent TERT promoter mutation in the majority of cancer types, and that lung cancer exhibited only the C228T mutation. In the present study, all cases with a TERT promoter mutation exhibited the C228T mutation and one case also exhibited a novel C247 (-143G $>\mathrm{T})$ mutation (Fig. 1A). The clinicopathological characteristics of patients with NSCLC and TERT promoter mutations are presented in Table I. TERT promoter mutation was statistically associated with lymph node invasion $(\mathrm{P}<0.001)$. TERT promoter mutation was observed more in patients with poor differentiation compared with patients with well-differentiated NSCLC $(0.7 \%$ vs. $7.7 \%)$; however, the difference was not statistically significant $(\mathrm{P}=0.060$; Table I).

TL was analyzed in only 99/188 patients with NSCLC due to a lack of DNA samples. The mean ( \pm standard deviation) TL in NSCLC was $3.04 \pm 0.30$, calculated as the ratio of TL in tumors to that of paired wild-type tissues. The mean TL of patients with NSCLC with and without TERT promoter mutations, was $1.82 \pm 0.49$ and $3.09 \pm 1.05$, respectively, exhibiting no statistically significant difference $(\mathrm{P}=0.282)$. To further investigate the association between TL and the clinicopathological features of patients with NSCLC, patients were categorized into two subgroups according to the mean value of the tumor/wild-type ratios (3.04). The clinicopathological characteristics of patients stratified to TL are summarized in Table II. TL was observed to be markedly decreased in males (72.2\%) compared with females (50.0\%) $(\mathrm{P}=0.058)$. However, TL exhibited a significant association with smokers compared with non-smokers (73.8 vs. $42.1 \%$; $\mathrm{P}=0.008$; Table II). To confirm the association between TL and smoking status, quantitative analysis was performed using Spearman's rank correlation coefficient (R) (Fig. 1B and C). The total pack-year of smoking was negatively associated with TL $(R=-0.210$; $\mathrm{P}=0.037)$. However, age did not demonstrate any significant association with $\mathrm{TL}(\mathrm{R}=0.015 ; \mathrm{P}=0.883)$. Other variables exhibited no significant association with TERT promoter mutation or TL.

Prognostic value of TERT promoter mutation and TL in $N S C L C$. We then assessed survival analysis to clarify any prognostic significance of TERT mutations and TL in NSCLC. The median follow-up of patients for survival analysis was 78.8 months (range, 1-123 months). Univariate survival analysis was performed using Kaplan-Meier estimators and demonstrated a decreased OS in patients with NSCLC with TERT promoter mutations compared with wild-type TERT (17.74 vs. 79.90 months; $\chi^{2}=13.25 ; \mathrm{P}<0.001$; Fig. 2A). However, TERT promoter mutation was not significantly associated with DFS (Fig. 2B). Survival analysis was also performed using TL and exhibited no significant prognostic value (OS, $\mathrm{P}=0.64$; $\mathrm{DFS}, \mathrm{P}=0.68$; data not shown). To evaluate whether TERT promoter mutation is an independent prognostic marker in patients with NSCLC, the data was 
Table I. Association between clinicopathological characteristics and TERT promoter mutation status in patients with non-small cell lung cancer.

TERT promoter status

\begin{tabular}{|c|c|c|c|c|}
\hline \multirow{2}{*}{ Clinicopathological characteristic } & \multirow[b]{2}{*}{$\mathrm{n}$} & & \multirow[b]{2}{*}{ P-value } \\
\hline & & Wild-type (\%) & Mutated (\%) & \\
\hline Total & 188 & $184(97.8)$ & $4(2.2)$ & \\
\hline Age, years & & & & 1.00 \\
\hline$<60$ & 89 & 87 (97.8) & $2(2.2)$ & \\
\hline$\geq 60$ & 99 & $97(98.0)$ & $2(2.0)$ & \\
\hline Gender & & & & 0.575 \\
\hline Male & 136 & $132(97.1)$ & $4(2.9)$ & \\
\hline Female & 50 & $50(100)$ & $0(0)$ & \\
\hline pT & & & & 1.00 \\
\hline $1 / 2$ & 157 & $153(97.5)$ & $4(2.5)$ & \\
\hline $3 / 4$ & 27 & $27(100)$ & $0(0)$ & \\
\hline $\mathrm{pN}$ & & & & $<0.001$ \\
\hline 0 & 155 & 153 (98.7) & $2(1.3)$ & \\
\hline 1 & 20 & $20(100)$ & $0(0)$ & \\
\hline 2 & 9 & $7(77.8)$ & $2(22.2)$ & \\
\hline Histological type & & & & 0.637 \\
\hline Squamous cell carcinoma & 97 & $94(96.9)$ & $3(3.1)$ & \\
\hline Adenocarcinoma & 90 & $89(98.9)$ & $1(1.1)$ & \\
\hline Differentiation status & & & & 0.060 \\
\hline Well/moderate & 146 & $145(99.3)$ & $1(0.7)$ & \\
\hline Poor/undifferentiated & 26 & $24(92.3)$ & $2(7.7)$ & \\
\hline Smoking & & & & 0.574 \\
\hline Yes/ever & 138 & $134(97.1)$ & $4(2.9)$ & \\
\hline Never & 49 & $49(100)$ & $0(0)$ & \\
\hline EGFR mutation status & & & & 1.00 \\
\hline Mutated & 37 & $37(100)$ & $0(0)$ & \\
\hline Wild-type & 110 & $109(99.1)$ & $1(0.9)$ & \\
\hline
\end{tabular}

Due to insufficient clinical data, not all patient characteristics have been presented. TERT, telomerase reverse transcriptase; pT, primary tumor grade; $\mathrm{pN}$, regional lymph node grade; EGFR, epidermal growth factor receptor.

further analyzed using the Cox proportional hazards model, following adjustment for possible confounders of survival (Table III). Following multivariate analysis, TERT promoter mutation $(\mathrm{OS}, \mathrm{HR}=13.16$; 95\% CI; 1.06-163.59; $\mathrm{P}=0.045$; and DFS, HR=9.99; 95\% CI, 0.87-114.18; $\mathrm{P}=0.064)$ exhibited potential value as an independent prognostic factor of OS. Histological type and EGFR mutation status also exhibited prognostic value, whereas TL did not demonstrate any statistical significance as an independent prognostic marker (OS, $\mathrm{HR}=2.08 ; 95 \% \mathrm{CI}, 0.79-5.92 ; \mathrm{P}=0.170 ; \mathrm{DFS}, \mathrm{HR}=1.17 ; 95 \%$ CI, 0.41-3.40; $\mathrm{P}=0.768)$.

\section{Discussion}

NSCLC, as a heterogeneous disease, has various outcomes even in patients with identical clinicopathological features. Identification of oncogenic driver mutations in NSCLC has promoted clinical treatment options, including targeted drugs.
For example, EGFR-targeted therapy using gefitinib increases survival results and prognosis of patients with NSCLC (15). To define molecular subtypes of NSCLC for personalized therapy, it is necessary to understand the driver genes and their molecular mechanisms, which currently remain unclear. Therefore, in the present study the clinicopathological characteristics and prognostic effects, of TERT promoter mutations and TL, were evaluated in patients with surgically resected NSCLC.

The results of the present study demonstrated that TERT promoter mutations were infrequent in patients with NSCLC; however, predicted poor OS following the construction of univariate and multivariate survival models. Previous studies reported that $\mathrm{C} 250(-146 \mathrm{C}>\mathrm{T})$ and $\mathrm{C} 228(-124 \mathrm{C}>\mathrm{T})$ were sites of frequent mutation, and that lung cancer exhibits only the C228T mutation (2/68 cell lines) (3). Recently, Li et al (16) identified no TERT promoter mutation in 174 NSCLC cases; however, Ma et al (17) reported it in $2.57 \%$ (12/467) of patients 
Table II. Association between clinicopathological characteristics and telomere length in patients with non-small cell lung cancer.

\begin{tabular}{|c|c|c|c|c|}
\hline \multirow[b]{2}{*}{ Clinicopathological characteristic } & \multirow[b]{2}{*}{$\mathrm{n}$} & \multicolumn{2}{|c|}{ Telomere length } & \multirow[b]{2}{*}{ P-value } \\
\hline & & Short $(\%)$ & Long $(\%)$ & \\
\hline Total & 99 & $67(67.7)$ & $32(32.3)$ & 1.00 \\
\hline Age, years & & & & 0.71 \\
\hline$<60$ & 53 & $35(66.0)$ & $18(34.0)$ & \\
\hline$\geq 60$ & 46 & $32(69.6)$ & $14(30.4)$ & \\
\hline Gender & & & & 0.058 \\
\hline Male & 79 & $57(72.2)$ & $22(27.8)$ & \\
\hline Female & 20 & $10(50.0)$ & $10(50.0)$ & \\
\hline $\mathrm{pT}$ & & & & 0.83 \\
\hline $1 / 2$ & 78 & $53(67.9)$ & $25(32.1)$ & \\
\hline $3 / 4$ & 17 & $12(70.6)$ & $5(29.4)$ & \\
\hline $\mathrm{pN}$ & & & & 0.78 \\
\hline 0 & 68 & $45(66.2)$ & $23(33.8)$ & \\
\hline 1 & 19 & $13(68.4)$ & $6(31.6)$ & \\
\hline 2 & 9 & $7(77.8)$ & $2(22.2)$ & \\
\hline Histological type & & & & 0.100 \\
\hline Squamous cell carcinoma & 61 & $45(73.8)$ & $16(26.2)$ & \\
\hline Adenocarcinoma & 38 & $22(57.9)$ & $16(42.1)$ & \\
\hline Differentiation & & & & 0.85 \\
\hline Well/moderate & 67 & $45(67.2)$ & $22(32.8)$ & \\
\hline Poor/undifferentiated & 17 & $11(64.7)$ & $6(35.3)$ & \\
\hline Smoking & & & & 0.008 \\
\hline Yes/ever & 80 & $59(73.8)$ & $21(26.3)$ & \\
\hline Never & 19 & $8(42.1)$ & $11(57.9)$ & \\
\hline EGFR mutation status & & & & 1.0 \\
\hline Mutated & 6 & $4(66.7)$ & $2(33.3)$ & \\
\hline Wild-type & 53 & $38(71.7)$ & $15(28.3)$ & \\
\hline
\end{tabular}

pT, primary tumor grade; pN, regional lymph node grade; EGFR, epidermal growth factor receptor.

Table III. Multivariate analyses of the prognostic values of various factors.

\begin{tabular}{|c|c|c|c|c|}
\hline \multirow[b]{2}{*}{ Factor } & \multicolumn{2}{|c|}{ Overall survival } & \multicolumn{2}{|c|}{ Disease-free survival } \\
\hline & HR $(95 \%$ CI $)$ & P-value & $\mathrm{HR}(95 \% \mathrm{CI})$ & P-value \\
\hline Age (>60 vs. $\leq 60$ years old $)$ & $1.39(0.58-3.33)$ & 0.455 & $1.64(0.64-4.22)$ & 0.304 \\
\hline Gender (female vs. male) & $1.47(0.43-5.05)$ & 0.534 & $1.63(0.42-6.38)$ & 0.478 \\
\hline Differentiation & $1.78(1.10-2.88)$ & 0.019 & $2.00(1.18-3.39)$ & 0.01 \\
\hline EGFR mutation status & $3.59(0.87-14.76)$ & 0.077 & $6.70(1.37-32.87)$ & 0.019 \\
\hline TERT promoter mutation status & $13.16(1.06-163.59)$ & 0.045 & $9.99(0.87-114.18)$ & 0.064 \\
\hline Telomere length (short vs. long) & $2.08(0.73-5.92)$ & 0.170 & $1.17(0.41-3.40)$ & 0.768 \\
\hline
\end{tabular}

EGFR, epidermal growth factor receptor; TERT, telomerase reverse transcriptase; HR, hazard ratio; CI, confidence interval.

with NSCLC. Previous reports identified certain novel TERT promoter mutations, which were associated with old age. In the present study, TERT promoter mutations were detected in $2.2 \%(4 / 188)$ of patients with NSCLC, which was consistent with previous studies (17). All cases with TERT promoter mutations exhibited a C228T mutation and one case exhibited the novel mutation, G247T (-143G $>$ T). Certain studies have reported novel mutations of the TERT promoter region; 


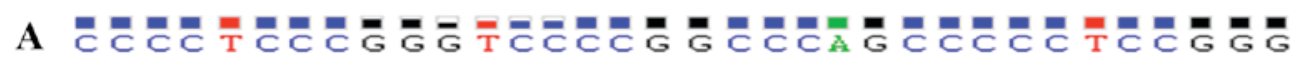
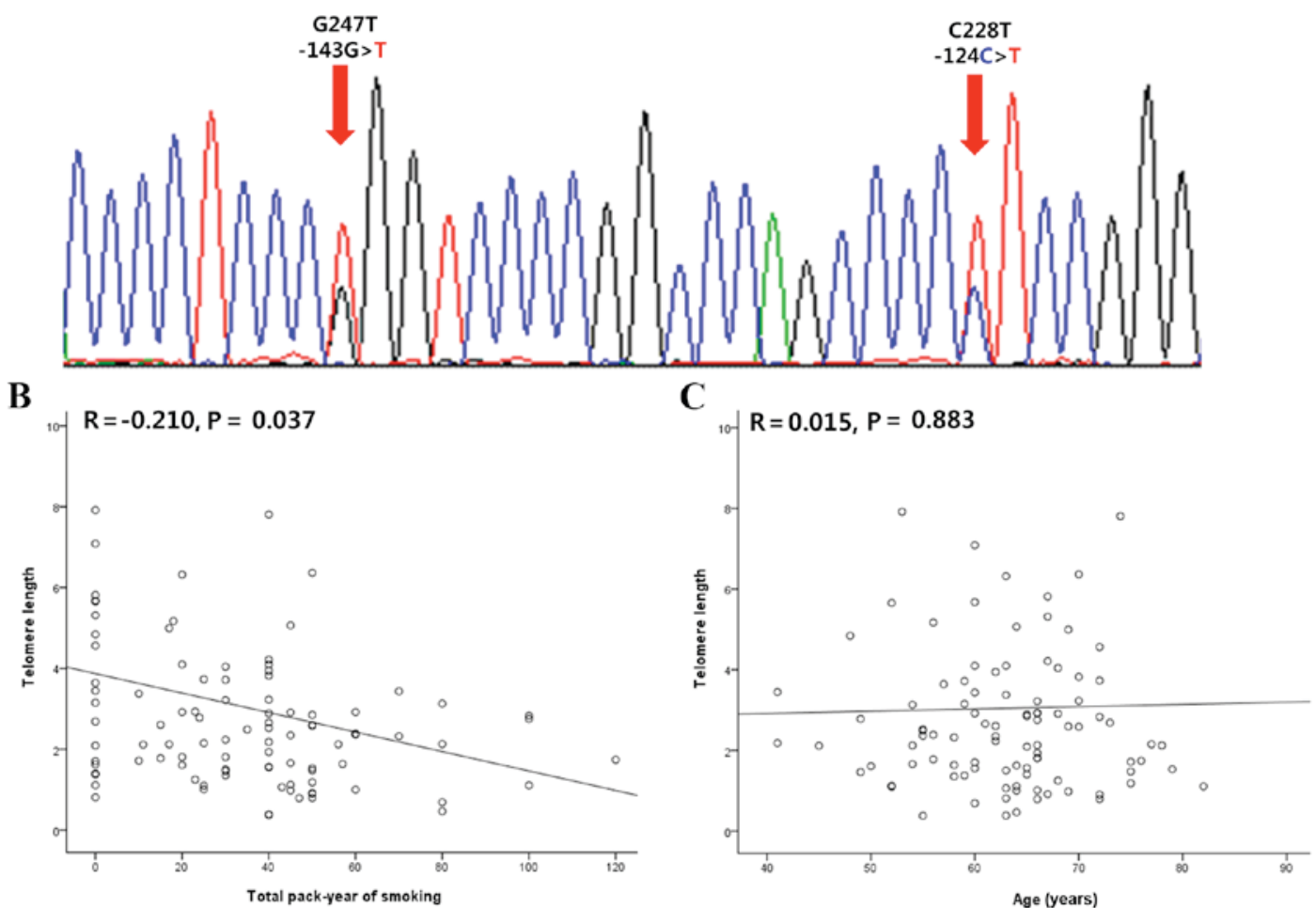

Figure 1. TERT promoter mutation status and telomere length in patients with non-small cell lung cancer. (A) Identification of C228T and G247T TERT promoter region mutations. (B) Negative association between telomere length and smoking status. (C) Association between telomere length and age. TERT, telomerase reverse transcriptase; $\mathrm{R}$, Spearman's rank correlation coefficient.
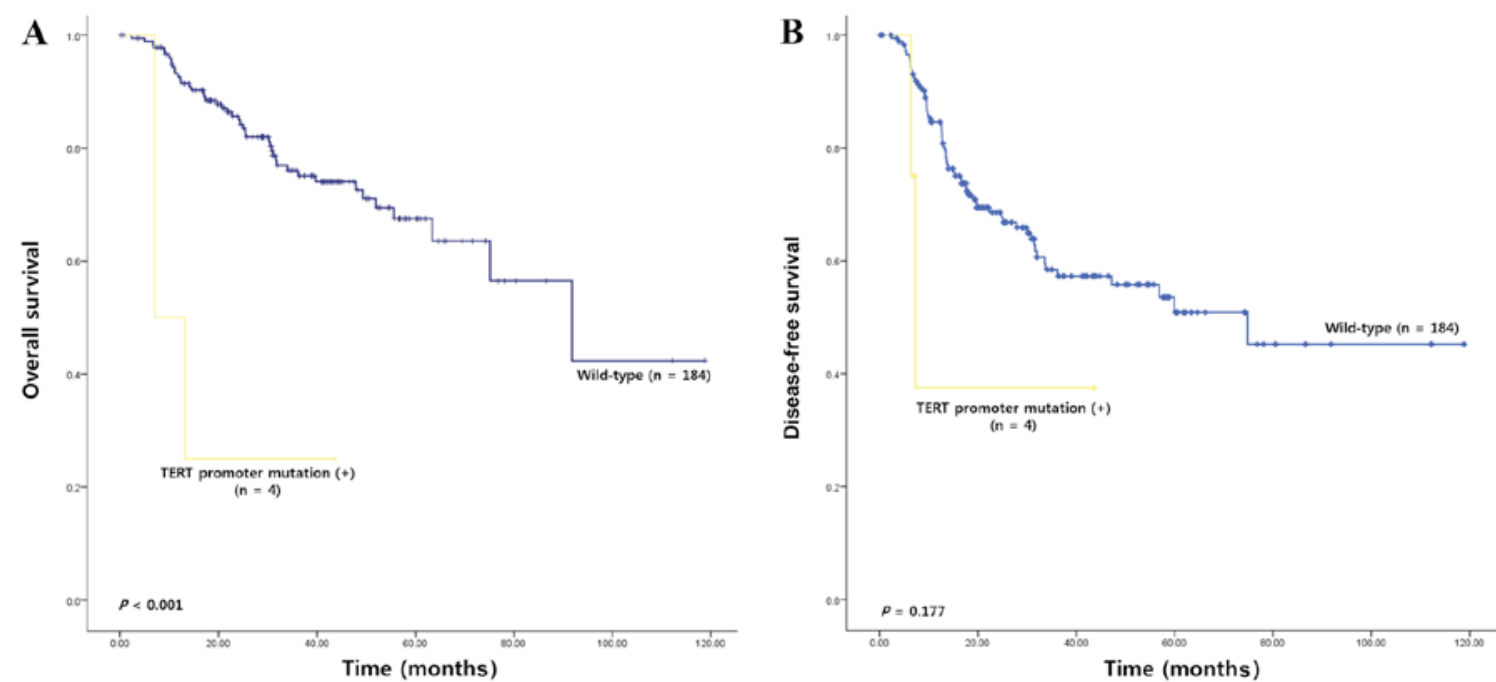

Figure 2. Survival analyses of 188 patients with non-small cell lung cancer. (A) Overall survival and (B) disease-free survival stratified by TERT promoter mutation status. TERT, telomerase reverse transcriptase.

however, the G247T mutation has not been reported in any type of cancer. In addition, TERT promoter mutations were associated with poor differentiation and lymph node invasion, which may predict a poor prognosis.

Consistent with these characteristics, survival of patients with NSCLC with TERT mutations was significantly decreased compared with patients without TERT promoter mutations, following univariate and multivariate analyses. Horn et al (4) previously demonstrated that TERT promoter mutations may create an E-twenty-six binding motif, resulting in the upregulation of TERT expression at the mRNA level in melanoma. Although alterations in TERT mRNA levels were not evaluated in the specimens of patients and the prognostic value of telomere-associated pathways remain unclear, the results of the present study suggested that TERT promoter mutations are an independent prognostic marker. Recent independent studies suggest that NSCLC patients with the 1st quartile (shortest) TL exhibit a poor outcome $(18,19)$. An 
age-dependent association between TERT promoter mutations and TL was also suggested in patients with thyroid cancer (5). As TL is hypothesized to be different depending on the presence or absence of TERT promoter mutations (3-5), TL was analyzed in the present study to elucidate the role of TERT promoter mutations and TL in NSCLC. The TL levels were not observed to be significantly associated with TERT promoter mutations. Conversely, TL exhibited an association with male gender and positive smoking status; however, these results may have originated from the high rate of smoking in Korean males (20). However, TL exhibited no statistically significant association with other clinicopathological characteristics or prognosis in Korean patients with NSCLC. Following stratification of variables, including TL, TERT promoter mutation status exhibited no prognostic significance (data not shown). Certain recent studies demonstrated no difference in TERT expression compared with the presence of TERT promoter mutations $(21,22)$. These reports indicate that tumor cells without TERT promoter mutations may have other mechanisms for activating TERT expression and changing TL. TERT promoter mutations may play a minor but independent role in lung carcinogenesis, inducing poor progression. To establish the true prognostic value of TERT promoter mutation, further study is required in larger cases. As EGFR mutation status was identified in the present study to be of prognostic significance in patients with NSCLC, analysis of EGFR mutation status in all samples is also required to elucidate the effect of TERT promoter mutation on lung carcinogenesis.

In conclusion, although the present study is limited by its retrospective design and small number of sample cases, the results demonstrate that TERT promoter mutations are infrequent in patients with NSCLC; however, are significantly associated with an unfavorable prognosis. These results provide novel insights into the consequences of TERT promoter mutations in patients with NSCLC and suggest their value as a prognostic marker in surgically resected NSCLC. The results of the present study warrant future large-scale studies to elucidate the underlying molecular mechanisms and to determine potential clinical utility.

\section{Acknowledgements}

This study was supported by grants of the Basic Science Research Program through the National Research Foundation of Korea (NRF) funded by the Ministry of Education (NRF-2014R1A6A3A04058057) and by the Korean Government (MSIP; No. 2014R1A5A2010008).

\section{References}

1. Yoo SS, Do SK, Choi JE, Lee SY, Cha SI, Kim CH and Park JY: TERT Polymorphism rs2853669 Influences on lung cancer risk in the korean population. J Korean Med Sci 30: 1423-1428, 2015.

2. Griewank KG, Murali R, Schilling B, Schimming T, Möller I, Moll I, Schwamborn M, Sucker A, Zimmer L, Schadendorf D and Hillen U: TERT promoter mutations are frequent in cutaneous basal cell carcinoma and squamouscell carcinoma. PLoS One 8: e80354, 2013.
3. Huang FW, Hodis E, Xu MJ, Kryukov GV, Chin L and Garraway LA: Highly recurrent TERT promoter mutations in human melanoma. Science 339: 957-959, 2013.

4. Horn S, Figl A, Rachakonda PS, Fischer C, Sucker A, Gast A, Kadel S, Moll I, Nagore E, Hemminki K, et al: TERT promoter mutations in familial and sporadic melanoma. Science 339: 959-961, 2013.

5. Liu X, Bishop J, Shan Y, Pai S, Liu D, Murugan AK, Sun H, El-Naggar AK and Xing M: Highly prevalent TERT promoter mutations in aggressive thyroid cancers. Endocr Relat Cancer 20: 603-610, 2013.

6. Killela PJ, Reitman ZJ, Jiao Y, Bettegowda C, Agrawal N, Diaz LA Jr, Friedman AH, Friedman H, Gallia GL, Giovanella BC, et al: TERT promoter mutations occur frequently in gliomas and a subset of tumors derived from cells with low rates of self-renewal. Proc Natl Acad Sci USA 110: 6021-6026, 2013.

7. Vinagre J, Almeida A, Pópulo H, Batista R, Lyra J, Pinto V, Coelho R, Celestino R, Prazeres H, Lima L, et al: Frequency of TERT promoter mutations in human cancers. Nat Commun 4: 2185, 2013.

8. Torre LA, Siegel RL and Jemal A: Lung Cancer Statistics. Adv Exp Med Biol 893: 1-19, 2016.

9. Travis WD, Travis LB and Devesa SS: Lung cancer. Cancer 75: 191-202, 1995.

10. Oser MG, Niederst MJ, Sequist LV and Engelman JA: Transformation from non-small-cell lung cancer to small-cell-lung cancer: Molecular drivers and cells of origin. Lancet Oncol 16: e165-e172, 2015.

11. Gertler R, Rosenberg R, Stricker D, Friederichs J, Hoos A, Werner M, Ulm K, Holzmann B, Nekarda H and Siewert JR: Telomere length and human telomerase reverse transcriptase expression as markers for progression and prognosis of colorectal carcinoma. J Clin Oncol 22: 1807-1814, 2004.

12. Svenson U, Nordfjäll K, Stegmayr B, Manjer J, Nilsson P, Tavelin B, Henriksson R, Lenner P and Roos G: Breast cancer survival is associated with telomere length in peripheral blood cells. Cancer Res 68: 3618-3623, 2008.

13. Liu T, Wang N, Cao J, Sofiadis A, Dinets A, Zedenius J, Larsson C and $\mathrm{Xu}$ D: The age- and shorter telomere-dependent TERT promoter mutation in follicular thyroid cell-derived carcinomas. Oncogene 33: 4978-4984, 2014.

14. Livak KJ and Schmittgen TD: Analysis of relative gene expression data using real-time quantitative PCR and the 2(-Delta Delta C(T)) method. Methods 25: 402-408, 2001.

15. Reck M: Gefitinib in the treatment of advanced non-small-cell lung cancer. Expert Rev Anticancer Ther 9: 401-412, 2009.

16. Li C, Hao L, Li Y, Wang S, Chen H, Zhang L, Ke B, Yin Y, Suo H, Sun B, et al: Prognostic value analysis of mutational and clinicopathological factors in non-small cell lung cancer. PLoS One 9: e107276, 2014.

17. Ma X, Gong R, Wang R, Pan Y, Cai D, Pan B, Li Y, Xiang J, Li H, Zhang J, et al: Recurrent TERT promoter mutations in non-small cell lung cancers. Lung Cancer 86: 369-373, 2014.

18. Fernández-Marcelo T, Gómez A, Pascua I, de Juan C, Head J, Hernando F, Jarabo JR, Calatayud J, Torres-García AJ and Iniesta P: Telomere length and telomerase activity in non-small cell lung cancer prognosis: Clinical usefulness of a specific telomere status. J Exp Clin Cancer Res 34: 78, 2015.

19. Sun B, Wang Y, Kota K, Shi Y, Motlak S, Makambi K, Loffredo CA, Shields PG, Yang Q, Harris CC and Zheng YL: Telomere length variation: A potential new telomere biomarker for lung cancer risk. Lung Cancer 88: 297-303, 2015.

20. Park JJ and Park HA: Prevalence of cigarette smoking among adult cancer survivors in Korea. Yonsei Med J 56: 556-562, 2015.

21. Chen YL, Jeng YM, Chang CN, Lee HJ, Hsu HC, Lai PL and Yuan RH: TERT promoter mutation in resectable hepatocellular carcinomas: A strong association with hepatitis $\mathrm{C}$ infection and absence of hepatitis B infection. Int J Surg 12: 659-665, 2014

22. Chiba K, Johnson JZ, Vogan JM, Wagner T, Boyle JM and Hockemeyer D: Cancer-associated TERT promoter mutations abrogate telomerase silencing. Elife 4: Jul 21, 2015 doi: 10.7554/eLife.07918. 